\title{
SIMULTANEOUS APPROXIMATION AND ALGEBRAIC INDEPENDENCE OF NUMBERS
}

\author{
BY WOLFGANG M. SCHMIDT
}

\author{
Communicated by Paul T. Bateman, April 14, 1962
}

1. The result. Write $\|\xi\|$ for the distance of a real number $\xi$ to the nearest integer. Given a polynomial $f$ in any number of variables, put $L(f)$ for the sum of the absolute values of its coefficients. Our main result is the following

TheOREM. Let $f\left(x_{1}, \cdots, x_{n}\right)$ be a nonzero polynomial with integral coefficients and of degree $d_{k}$ in $x_{k}(k=1, \cdots, n)$. Let $Q>0$ and $q \neq 0$ be integers and $\xi_{1}, \cdots, \xi_{n}$ reals satisfying

$$
|q| \leqq Q, \quad\left|\xi_{k} q\right| \leqq Q \quad(k=1, \cdots, n)
$$

and

(2) $\left\|\xi_{k} q\right\| \leqq\left\|\xi_{1} q\right\|\left\|^{d_{1}} \cdots\right\| \xi_{k-1} q \|^{d_{k-1}} Q^{1-d_{1}-\cdots-d_{n}}\left[d_{k} 2^{d_{k}+\cdots+d_{n}+k} L(f)\right]^{-1}$

Then

$$
(k=1,2, \cdots, n){ }^{1}
$$

$$
\left|f\left(\xi_{1}, \cdots, \xi_{n}\right)\right| \geqq\left\|\xi_{1} q\right\|\left\|^{d_{1}} \cdots\right\| \xi_{n} q\|\|_{d_{n}} q^{-d_{1}-\cdots-d_{n} 2^{-n}} .
$$

Generalizing a well-known result of Liouville [1] for $n=1$ (for further references, see [4]), we obtain a

SUFFICIENT CONDITION FOR ALGEBRAIC INDEPENDENCE. Let $\xi_{1}, \cdots, \xi_{n}$ be an $n$-tuple of reals such that to every $d>0$ there is an integer $q$ with

$$
0<\left\|\xi_{k} q\right\|<\left(\left\|\xi_{1} q\right\| \cdots\left\|\xi_{k-1} q\right\|\right)^{d} q^{1-n d} \quad(k=1,2, \cdots, n) .^{1}
$$

Then $\xi_{1}, \cdots, \xi_{n}$ are algebraically independent (over the rationals).

This is true since (2) can be satisfied with

$$
Q \geqq \max \left(q,\left|\xi_{1} q\right|, \cdots,\left|\xi_{n} q\right|\right)
$$

and for every $f$, if (4) can be satisfied for every $d$.

For example, the numbers $\xi_{1}, \xi_{2}, \ldots$ defined by

$$
\xi_{k}=\sum_{t=1}^{\infty} 2^{-(k t) !} \quad(k=1,2, \cdots)
$$

are independent. ${ }^{2}$ It suffices to show that $\xi_{1}, \cdots, \xi_{n}$ are independent

\footnotetext{
${ }^{1}$ For $k=1$, the product of the $\left\|\xi_{i} q\right\|$ 's means 1 .

${ }^{2} \mathrm{~A}$ similar system of algebraically independent numbers was given by von Neumann [3]. Actually von Neumann's system has the power of the continuum.

Added in proof. Using our criterion and the sequence $q_{t}=2^{\left(t^{t}\right)}$ one can show that

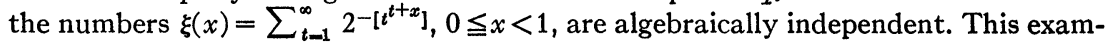
ple (with $0<x \leqq 1$ ) is due to H. Kneser (Bull. Soc. Math. Belg. 12 (1960), 23-27).
} 
for fixed $n$. Take a positive integer $h$ with $n ! \mid h$ and put $q_{h}=2^{h !}$. Then

which yields

$$
\left\|\xi_{k} q_{h}\right\|=2^{h !} \sum_{t=h / k+1}^{\infty} 2^{-(k t) !} \quad(k=1, \cdots, n),
$$

and

$$
2^{h !-(h+k) !}<\left\|\xi_{k} q_{h}\right\|<2^{1+h !-(h+k) !} \quad(k=1, \cdots, n)
$$

$$
\left(\left\|\xi_{1} q_{k}\right\| \cdots\left\|\xi_{k-1} q_{k}\right\|\right)^{d} q_{h}^{-n d}>2^{-n d(h+k-1) !-n d h !} \quad(k=1, \cdots, n) .
$$

Hence $q_{h}$ for $h>h(d)$ satisfies our condition, and the assertion is proved.

2. The proof. Let $Q>0, q \neq 0, p_{1}, \cdots, p_{n}$ be integers such that

$$
|q| \leqq Q, \quad\left|p_{1}\right| \leqq Q, \cdots,\left|p_{n}\right| \leqq Q .
$$

Let $f$ be a polynomial as described in the theorem, and put $L_{Q}(f)$ $=L\left(f\left(Q x_{1}, \cdots, Q x_{n}\right)\right)$.

We define now recursively a set of $2 n+1$ polynomials

$$
f_{n}, g_{n}, \cdots, f_{1}, g_{1}, f_{0}
$$

as follows:

$$
f_{n}\left(x_{1}, \cdots, x_{n}\right)=q^{d_{1}+\cdots+d_{n}} f\left(x_{1} / q, \cdots, x_{n} / q\right) ;
$$

if $f_{k}\left(x_{1}, \cdots, x_{k}\right)$ is already defined, let $\delta_{k}$ be the largest non-negative integer such that $\left(x_{k}-p_{k}\right)^{\delta_{k}} \mid f_{k}\left(x_{1}, \cdots, x_{k}\right)$, and then put

$$
g_{k}\left(x_{1}, \cdots, x_{k}\right)=\left(x_{k}-p_{k}\right)^{-\delta_{k}} f_{k}\left(x_{1}, \cdots, x_{k}\right)
$$

and

$$
f_{k-1}\left(x_{1}, \cdots, x_{k-1}\right)=g_{k}\left(x_{1}, \cdots, x_{k-1}, p_{k}\right) .
$$

It is clear that these polynomials are $\neq \equiv 0$.

From the definition,

$$
L_{Q}\left(f_{n}\right)=\sum_{i_{1}} \cdots \sum_{i_{n}}\left|a_{i_{1}} \cdots i_{n}\right| q^{d_{1}+\cdots+d_{n}-i_{1}-\cdots-i_{n}} Q^{i_{1}+\cdots+i_{n}}
$$

and hence

$$
L_{Q}\left(f_{n}\right) \leqq Q^{d_{1}+\cdots+d_{n}} L(f) .
$$

Assume next that, say,

$$
f_{k}\left(x_{1}, \cdots, x_{k}\right)=\sum_{i_{1}=0}^{d_{1}} \cdots \sum_{i_{k}=0}^{d_{k}} a(k)_{i_{1}} \cdots i_{k} x_{1}^{i_{1}} \cdots x_{k}^{i_{k}}
$$

and 
1962] SIMULTANEOUS APPROXIMATION AND INDEPENDENCE OF NUMBERS 477

$$
g_{k}\left(x_{1}, \cdots, x_{k}\right)=\sum_{i_{1}=0}^{d_{1}} \cdots \sum_{i_{k}=0}^{d_{k}} b(k)_{i_{1}} \cdots i_{k} x_{1}^{i_{1}} \cdots x_{k}^{i_{k}} .
$$

It is then clear from the definition of $g_{k}$ in terms of $f_{k}$ that, for every choice of $i_{1}, \cdots, i_{k-1}$,

$$
\sum_{i_{k}=0}^{d_{k}} a(k)_{i_{1}} \ldots i_{k} x_{k}^{i_{k}} \equiv\left(x_{k}-p_{k}\right)^{\delta_{k}} \sum_{i_{k}=0}^{d_{k}} b(k)_{i_{1}} \ldots i_{k} x_{k}^{i_{k}} .
$$

An inequality of Mahler [2] states that

$$
\prod_{\sigma=1}^{s} L\left(g_{\sigma}\right) \leqq 2^{d} L\left(\prod_{\sigma=1}^{s} g_{\sigma}\right)
$$

where $g_{1}(x), \cdots, g_{s}(x)$ are polynomials in one variable and $d$ is the degree of their product. Putting $s=2, g_{1}(x)=\left(Q x-p_{k}\right)^{\delta_{k}}, g_{2}(x)$ $=\sum_{i_{k}=0}^{d_{k}} b(k)_{i_{1}} \ldots i_{k} Q^{i_{k}} x^{i_{k}}$ and applying this inequality, we find

$$
\sum_{i_{k}=0}^{d_{k}}\left|b(k)_{i_{1}} \ldots i_{k}\right| Q^{i_{k}} \leqq 2^{d_{k}} \sum_{i_{k}=0}^{d_{k}}\left|a(k)_{i_{1}} \ldots i_{k}\right| Q^{i_{k}} .
$$

This we multiply by $Q^{i_{1}+\cdots+i_{k-1}}$ and sum over all allowed $i_{1}, \cdots, i_{k-1}$. The result so obtained is that ${ }^{3}$

$$
L_{Q}\left(g_{k}\right) \leqq 2^{d_{k}} L_{Q}\left(f_{k}\right) .
$$

Successive application of (5), (6), and the easily obtained formula

$$
L_{Q}\left(f_{k-1}\right) \leqq L_{Q}\left(g_{k}\right)
$$

yields

$$
L_{Q}\left(g_{k}\right) \leqq 2^{d_{k}+\cdots+d_{n}} Q^{d_{1}+\cdots+d_{n}} L(f) \quad(k=n, \cdots, 1) .
$$

Let now $\epsilon_{1}, \cdots, \epsilon_{n}$ be real numbers, and assume that the former inequalities for $q$ and $p_{k}$ are replaced by the stronger formulae

$$
|q| \leqq Q, \quad\left|p_{k}\right| \leqq Q, \quad\left|p_{k}+\epsilon_{k}\right| \leqq Q \quad(k=1, \cdots, n) .
$$

It follows then from the mean value theorem that

$$
\begin{aligned}
\Delta_{k} \equiv & g_{k+1}\left(p_{1}+\epsilon_{1}, \cdots, p_{k}+\epsilon_{k}, p_{k+1}+\epsilon_{k+1}\right) \\
& -g_{k+1}\left(p_{1}+\epsilon_{1}, \cdots, p_{k}+\epsilon_{k}, p_{k+1}\right) \\
= & \left.\epsilon_{k+1} \frac{\partial}{\partial x_{k+1}} g_{k+1}\left(p_{1}+\epsilon_{1}, \cdots, p_{k}+\epsilon_{k}, x_{k+1}\right)\right|_{x_{k+1}=p_{k+1}+\theta \epsilon_{k+1}},
\end{aligned}
$$

${ }^{3}$ A reader not familiar with Mahler's inequality should have no difficulty proving $L_{Q}\left(g_{k}\right) \leqq c_{1}(f) L_{Q}\left(f_{k}\right)$. This yields a theorem where the expression in brackets in (2) is replaced by $c_{2}(f)$, and it yields our condition for independence. 
where $\theta$ is a certain constant satisfying $0<\theta<1$, so that also $\left|x_{k+1}\right|$ $\leqq Q$. Thus we find that

and hence by (8),

$$
\left|\Delta_{k}\right| \leqq\left|\epsilon_{k+1}\right| d_{k+1} Q^{-1} L_{Q}\left(g_{k+1}\right)
$$

$$
\left|\Delta_{k}\right| \leqq\left|\epsilon_{k+1}\right| Q^{d_{1}+\cdots+d_{n}-1} d_{k+1} 2^{d_{k+1}+\cdots+d_{n}} L(f) .
$$

Assume now that $\left|\epsilon_{k}\right| \leqq 1(k=1, \cdots, n)$ and that

$$
\begin{array}{r}
\left|\epsilon_{k}\right| \leqq\left|\epsilon_{1}\right|^{d_{1}} \cdots\left|\epsilon_{k-1}\right|^{d_{k-1}} Q^{1-d_{1}-\cdots-d_{n}}\left[d_{k} 2^{d_{k}+\cdots+d_{n}+k} L(f)\right]^{-1} \\
(k=1, \cdots, n) .
\end{array}
$$

We are going to show

$$
\begin{array}{r}
\left|f_{k}\left(p_{1}+\epsilon_{1}, \cdots, p_{k}+\epsilon_{k}\right)\right| \geqq\left|\epsilon_{1}\right|^{d_{1}} \cdots\left|\epsilon_{k}\right|^{d_{k} 2^{-k}} \\
(k=0,1, \cdots, n) .
\end{array}
$$

The formula is true for $k=0$ since $\left|f_{0}\right| \geqq 1$. If it is true for some $k<n$, then by (10) and (11),

$$
\begin{gathered}
\left|g_{k+1}\left(p_{1}+\epsilon_{1}, \cdots, p_{k+1}+\epsilon_{k+1}\right)-f_{k}\left(p_{1}+\epsilon_{1}, \cdots, p_{k}+\epsilon_{k}\right)\right| \\
\leqq 2^{-1}\left|f_{k}\left(p_{1}+\epsilon_{1}, \cdots, p_{k}+\epsilon_{k}\right)\right|
\end{gathered}
$$

hence

$$
\begin{aligned}
& \left|f_{k+1}\left(p_{1}+\epsilon_{1}, \cdots, p_{k+1}+\epsilon_{k+1}\right)\right| \\
& \quad=\left|\epsilon_{k+1}\right| \delta_{k+1}\left|g_{k+1}\left(p_{1}+\epsilon_{1}, \cdots, p_{k+1}+\epsilon_{k+1}\right)\right| \\
& \quad \geqq 2^{-k-1}\left|\epsilon_{1}\right|^{d_{1}} \cdots\left|\epsilon_{k}\right|^{d_{k}}\left|\epsilon_{k+1}\right|^{\delta_{k+1}},
\end{aligned}
$$

and (12) is true for $k+1$.

The proof of the theorem is now immediate. Assume (1) and (2) to be satisfied. Put $\xi_{k} q=p_{k}+\epsilon_{k}(k=1, \cdots, n)$, where $p_{k}$ is integral and $\left|\epsilon_{k}\right|=\left\|\xi_{k} q\right\|$. Then (1) yields (9) because $Q$ and the $p_{k}$ 's are integral, and (2) gives (11). We obtain

$$
\begin{aligned}
\left|q^{d_{1}+\cdots+d_{n}} f\left(\xi_{1}, \cdots, \xi_{n}\right)\right| & =\left|f_{n}\left(p_{1}+\epsilon_{1}, \cdots, p_{n}+\epsilon_{n}\right)\right| \\
& \geqq\left|\epsilon_{1}\right|^{d_{1}} \cdots\left|\epsilon_{n}\right| d_{n} 2^{-n},
\end{aligned}
$$

thereby proving our theorem.

I am grateful to Professor Mahler for simplifying my original proof.

\section{REFERENCES}

1. J. Liouville, Sur les classes très etendus de quantites dont la valeur n'est algebriques, etc., C. R. Acad. Sci. Paris 18 (1844), 883-885, 910-911.

2. K. Mahler, An application of Jensen's formula to polynomials, Mathematika 7 (1960), 98-100.

3. J. von Neumann, Ein System algebraisch unabhängiger Zahlen, Ann. of Math. 99 (1928), 134-141.

4. Th. Schneider, Einführung in die transzendenten Zahlen, Springer, Berlin, 1957.

Columbia University 